\title{
Early effects of transcatheter aortic valve implantation and aortic valve replacement on myocardial function and aortic valve hemodynamics: Insights from cardiovascular magnetic resonance imaging
}

Gareth Crouch, MBBS, ${ }^{\text {a,b }}$ Jayme Bennetts, MBBS, ${ }^{\text {a,b }}$ Ajay Sinhal, MD, ${ }^{c}$ Phillip J. Tully, PhD, ${ }^{b}$ Darryl P. Leong, PhD, ${ }^{a}$ Craig Bradbrook, MRS, ${ }^{c}$ Amy L. Penhall, BSc, ${ }^{c}$ Carmine G. De Pasquale, PhD, ${ }^{\mathrm{a}, \mathrm{c}}$ Adhiraj Chakrabarty, MBBS, ${ }^{\mathrm{c}, \mathrm{d}}$ Robert A. Baker, PhD, ${ }^{\mathrm{a}, \mathrm{b}}$ and Joseph B. Selvanayagam, DPhil ${ }^{\mathrm{a}, \mathrm{c}, \mathrm{d}}$

Objectives: There remains a paucity of mechanistic data on the effect of transcatheter aortic valve implantation (TAVI) on early left and right ventricular function and quantitative aortic valve regurgitation. We sought to assess and compare the early effects on myocardial function and aortic valve hemodynamics of TAVI and aortic valve replacement (AVR) using serial cardiovascular magnetic resonance (CMR) imaging and echocardiography.

Methods: A prospective comparison study of 47 patients with severe aortic stenosis undergoing either TAVI $(\mathrm{n}=26)$ or high-risk AVR $(\mathrm{n}=21)$. CMR (for left ventricle/right ventricle function, left ventricular mass, left atrial volume, and aortic regurgitation) was carried out before the procedure and early postprocedure $(<14$ days).

Results: Groups were similar with respect to Society of Thoracic Surgeons score (TAVI, 7.7 vs AVR, 5.9; $P=.11$ ). Preoperative left ventricular (TAVI, $69 \% \pm 13 \%$ vs AVR, $73 \% \pm 10 \% ; P=.10$ ) and right ventricular (TAVI, $61 \% \pm 11 \%$ vs AVR, $59 \% \pm 8 \% ; P=.5$ ) ejection fractions were similar. Postoperative left ventricular ejection fraction was preserved in both groups. In contrast, decline in right ventricular ejection fraction was more significant in the TAVI group $(61 \%-54 \%$ vs $59 \%-58 \% ; P=.01)$. Postprocedure aortic regurgitant fraction was significantly greater in the TAVI group ( $16 \%$ vs $4 \% ; P=.001)$, as was left atrial size (110 vs $84 \mathrm{~mL} ; P=.02)$. Further analysis revealed a significant relationship between the increased aortic regurgitant fraction and greater left atrial size $(P=.006)$, and a trend toward association between the decline in right ventricle dysfunction and increased postprocedure aortic regurgitation $(P=.08)$.

Conclusions: There was no significant difference in early left ventricular systolic function between techniques. Whereas right ventricle systolic function was preserved in the AVR group, it was significantly impaired early after TAVI, possibly reflecting a clinically important pathophysiologic consequence of paravalvular aortic regurgitation. (J Thorac Cardiovasc Surg 2015;149:462-70)

Aortic stenosis is an increasingly common condition associated with significant morbidity and mortality, and consequent public health burden. ${ }^{1}$ Although surgical aortic valve replacement (AVR) has been the mainstay of treatment over

From Flinders University, ${ }^{\text {a }}$ Adelaide, Australia; Departments of Cardiothoracic Surgery $^{\mathrm{b}}$ and Cardiology, ${ }^{\mathrm{c}}$ Flinders Medical Centre, Adelaide, Australia; and the South Australian Health and Medical Research Institute, ${ }^{\mathrm{d}}$ Adelaide, Australia.

Supported by unencumbered research grants from St Jude Medical and Edwards Lifesciences.

Disclosures: Ajay Sinhal reports consulting fees from Edwards and lecture fees from Medtronic and Astra Zeneca. Carmine G. De Pasquale reports consulting fees from Novartis and lecture fees from Servier. Jayme Bennetts is an advisor for Medtronic. All other authors have nothing to disclose with regard to commercial support.

Received for publication Sept 3, 2014; revisions received Oct 7, 2014; accepted for publication Oct 11, 2014; available ahead of print Nov 22, 2014.

Address for reprints: Joseph B. Selvanayagam, DPhil, Department of Cardiology, Flinders Medical Centre, Flinders Dr, Bedford Park, South Australia, 5042

Australia (E-mail: joseph.selva@health.sa.gov.au).

0022-5223/\$36.00

Copyright (C) 2015 by The American Association for Thoracic Surgery

http://dx.doi.org/10.1016/j.jtcvs.2014.10.064 the past 4 decades, transcatheter aortic valve implantation (TAVI) has emerged as an attractive option, especially in patients with high or prohibitive surgical risk. ${ }^{2-6}$ Despite widespread clinical use there remains limited data on hemodynamics early post-TAVI, which may have important prognostic implications.

Paravalvular aortic regurgitation (PAR) in particular has emerged as a potentially important determinant of short- and medium-term clinical outcomes after TAVI ${ }^{7}$; however, there is a paucity of mechanistic data on the effect of TAVI-related aortic regurgitation (AR) on ventricle structure and function. Furthermore, the incidence, extent, and temporal sequence of myocardial reversible and irreversible injury are poorly characterized after both TAVI and AVR. ${ }^{8,9}$ The few studies to date that have examined early left ventricle (LV) function and aortic valve hemodynamic effects following TAVI have used transthoracic echocardiography (TTE), which has 

Abbreviations and Acronyms
ANOVA $=$ analysis of variance
$\mathrm{AR}=$ aortic regurgitation
AVR $=$ aortic valve replacement
CMR = cardiovascular magnetic resonance
LGE = late gadolinium enhancement
$\mathrm{LV} \quad=$ left ventricle
LVEDV $=$ left ventricular end-diastolic volume
LVESV = left ventricular end-systolic volume
PAR = paravalvular aortic regurgitation
QCA = quantitative coronary angiography
$\mathrm{RV} \quad=$ right ventricle
TAVI = transcatheter aortic valve implantation
TTE $=$ transthoracic echocardiography

substantial limitations related to image quality and sensitivity, especially in a postprocedure setting. ${ }^{10}$ Further, TTE is severely restricted in right ventricle (RV) assessment, particularly in the perioperative setting. ${ }^{11}$

High-resolution cardiovascular magnetic resonance (CMR) imaging is a safe, noninvasive technique that allows serial assessment of myocardial function and tissue characterization in the periprocedure setting. ${ }^{9,12-16}$ Given its 3-dimensional nature and superior signal to noise ratio, cine CMR is highly superior to 2-dimensional echocardiography and has become the gold standard investigation for measurement of LV/RV volumes, mass, and function of both normal and abnormal ventricles. ${ }^{9,17-19}$ Finally, it allows quantitative assessment of both native and prosthetic aortic valve flow dynamics, including parameters such as peak velocity and regurgitation volume. ${ }^{20}$ Using this highly accurate and reproducible technique, in a single-center prospective cohort trial, we compared the extent of perioperative LV and RV myocardial injury in patients undergoing TAVI with those undergoing high-risk AVR. Furthermore, we sought to characterize the association between postprocedure AR (as assessed by CMR imaging) with effects on the RV and LV. We hypothesized that TAVI would result in significantly less LV and RV myocardial stunning compared with AVR. Furthermore, we speculated that the occurrence of PAR would be correlated with worse myocardial function following the procedure.

\section{METHODS}

\section{Ethics}

This study was approved by the Human Research Ethics Committee of Flinders Medical Centre (approval No. 237.11) and conducted in accordance with the Declaration of Helsinki. All patients gave written informed consent.

\section{Patient Selection}

Transcatheter aortic valves remain an investigational device in Australia and are approved for use in patients deemed inoperable or high-risk for
AVR. Patients with severe symptomatic aortic stenosis referred for intervention were assessed by the heart team, taking into consideration age, comorbidities, risk scores, and frailty. A clinical decision then determined whether the individual proceeded to AVR or TAVI. Patients undergoing TAVI who were included were all from the high-risk cohort, with inoperable patients excluded.

To limit bias, a high-risk cohort of patients with AVR was selected. Inclusion criteria were EuroSCORE $>12$ or Society of Thoracic Surgeons risk score $>4$, age $>70$ years, and subjective frailty assessment. ${ }^{21}$ Additionally, patients with a preprocedure LV ejection fraction $<45 \%$ were excluded to maintain homogeneity in periprocedural functional assessment.

\section{Study Protocol}

Consenting patients who met selection criteria had preprocedural investigation within 14 days of their procedure. This included biochemistry, echocardiography, and CMR. Postprocedure, patients had echocardiography and CMR within 14 days (Figure 1). All patients underwent preprocedure coronary angiography.

\section{AVR and TAVI Techniques}

All open surgery was performed by experienced cardiothoracic surgeons. Techniques were similar, being standard median sternotomy and cardiopulmonary bypass with diastolic arrest achieved by antegrade tepid blood cardioplegia. Three tissue valve prostheses were used: Medtronic Mosaic (Medtronic Inc, Minneapolis, Minn), St Jude Medical Epic (St Jude Medical Inc, St Paul, Minn), and Trifecta (St Jude Medical Inc).

Transcatheter valve procedures were performed by an interventional cardiologist (A.S.) and cardiac surgeon (J.B.) with implantation of 80 valves before this study. All TAVIs were performed using combined angiography and transoesophegeal echocardiography guidance. All procedures used the Edwards Sapien XT prosthesis (Edwards Lifesciences, Irvine, Calif) deployed transfemorally.

\section{CMR Imaging Protocol}

Patients were studied in a 1.5-T CMR imaging scanner (Siemens Aera, Munich, Germany), and steady-state free precession cine images (TE/TR $1.5 / 3.0 \mathrm{~ms}$, flip angle $60^{\circ}$ ) were acquired in 2 long-axis and 8 to 10 short-axis views. The acquisition of short-axis views began $1 \mathrm{~cm}$ below the level of the mitral valve insertion plane and continued in $1-\mathrm{cm}$ increments through the LV and RV.

Forward and regurgitant aortic flows were measured using through-plane phase-contrast velocity mapping (free breathing, retrospective gating). The image plane was placed approximately $0.5 \mathrm{~cm}$ above the aortic valve at end-diastole, and maintained throughout the cardiac cycle. Commercially available gadolinium-based contrast agent (Gadovist 1.0, Gadobutrol; Bayer Healthcare, Berlin, Germany) was given to those patients with a glomerular filtration rate $>45 \mathrm{~mL} / \mathrm{min} / \mathrm{m}^{2}$. Images were acquired after a 6-minute delay with the use of an inversion-recovery segmented gradient echo sequence. Late gadolinium enhancement (LGE) images were acquired in identical long- and short-axis planes to the cine images, except for the most apical short-axis slice, which was excluded. Regional wall motion analysis was performed by two blinded observers using a 16 segment American Heart Association model and the following 0 to 4 scale, where $0=$ normal, $1=$ mildly hypokinetic, $2=$ severely hypokinetic, $3=$ akinetic, and $4=$ dyskinetic.

\section{Postprocessing Analysis}

A standardized method for analyzing and calculating LV and RV volumes was used. These methods along with their reproducibility have been previously published. ${ }^{22}$ Analysis was performed using commercially available software CMR42 (Circle Cardiovascular Imaging, Calgary, Canada). Left atrial volume was measured using the biplane area-length method using CMR 2- and 4-chamber views. ${ }^{23}$ Preoperative CMR LV short axis images were analyzed for regional wall motion abnormalities using a standard 17 


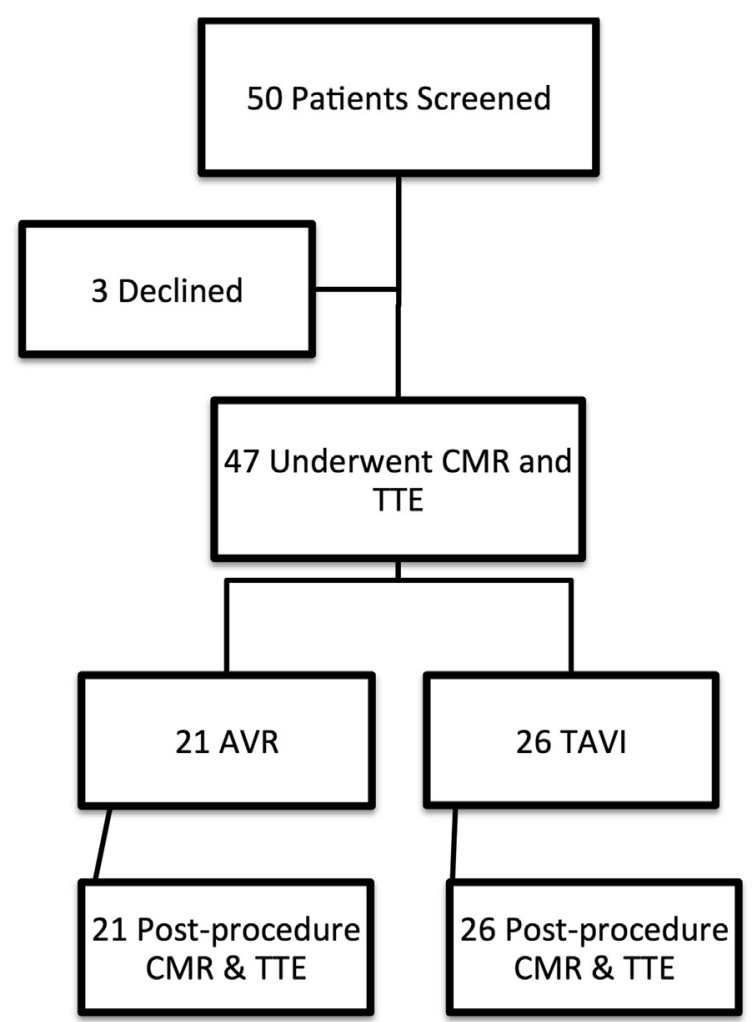

FIGURE 1. Study pathway. $C M R$, Cardiovascular magnetic resonance imaging; TTE, transthoracic echocardiography; $A V R$, aortic valve replacement; TAVI, transcatheter aortic valve implantation.

segment American Heart Association model grading wall motion from 0 to 4 according to previously published criteria. ${ }^{14}$ Areas of myocardial infarction were quantified using the 5 standard deviation method. ${ }^{24}$

\section{Echocardiography Protocol}

TTE was performed before and after the procedure concurrent with CMR, to examine LV diastolic function. Vivid E9 ultrasound machines were used and results were analyzed offline using EchoPAC PC Version 7 (General Electric-Vingmed Ultrasound, Milwaukee, Wis). Transmitral E- and A-wave velocities were measured using pulse wave Doppler at the mitral valve leaflet tips. Mitral $\mathrm{E}^{\prime}$ was measured using pulse wave Tissue Doppler by positioning the sample volume at the septal mitral annulus.

\section{Coronary Angiography Analysis}

Severity of coronary artery lesions was quantified using quantitative coronary angiography (QCA) by automated software and assessed visually when not suitable for QCA. A cutoff of $>50 \%$ diameter stenosis was used to classify single, double, or triple vessel disease. Any lesion ( $>70 \%$ diameter stenosis by QCA) that was not revascularized was labelled incompletely revascularized.

\section{Statistical Analysis}

Values are expressed as mean \pm standard deviation or median (interquartile range) as appropriate. All values were checked for normality and the following transformations were made: LV ejection fraction before and after the procedure (inverse), left ventricular end-diastolic volume (LVEDV) (square root), left ventricular end-systolic volume (LVESV) $(\log )$, RV ejection fraction (RVEF) (square root), right ventricular enddiastolic volume (RVEDV) (square root), right ventricular end-systolic volume (RVESV) (square root), brain natriuretic peptide (log), and regurgitation fraction $(\log )$.

Descriptive comparisons (TAVI vs AVR) were made with independent samples $t$ test and the $\chi^{2}$ or Fisher exact test as appropriate. Changes in indices of cardiac function were evaluated with repeated measures analysis of variance (ANOVA). The ANOVA model specified main effects for time, between group effects (TAVI vs AVR), and interaction effect time by group. ANOVA findings were corroborated using linear mixed effects models, with patient identity incorporated as a random effect. Additionally, in ancillary analysis adjustments were made for propensity score to reduce bias due to the nonrandomized TAVI allocation. Calculated with logistic regression, the propensity score determines the likelihood that a patient was treated with TAVI based on demographic and comorbid conditions. Given the small numbers, Society of Thoracic Surgeons risk score was chosen as the single characteristic that represented all factors influencing preference for TAVI (eg, age, reoperation, LV function, chronic obstructive pulmonary disease, and cerebrovascular accident). Given the study focus on RV systolic function, change in RV ejection fraction was analyzed with repeated measures ANOVA in a full-factorial model and consideration was given to a number of indices selected a priori for their influence on RV ejection fraction (age, N-terminal pro B-type natriuretic peptide, postprocedure LV ejection fraction, and AR). Covariates were entered as interaction terms (covariate $\times$ group $\times$ time) in addition to main and between group effects. The area under the curve for serial high-sensitivity troponin $\mathrm{T}$ measurements was calculated according to Matthews and colleagues ${ }^{25}$ and analyzed with Mann-Whitney $U$ test. The difference in proportion of patients with $\mathrm{N}$-terminal pro B-type natriuretic peptide values exceeding normal reference range of $125 \mathrm{ng} / \mathrm{L}$ was analyzed with Fisher exact test. Statistical analyses were performed with SPSS version 20.00 (IBM-SPSS Inc, Armonk, NY) and STATA (StataCorp, College Station, Tex).

\section{RESULTS}

\section{Baseline Clinical Characteristics}

A total of 48 patients were recruited, with 1 exclusion due pacemaker implantation, leaving 47 patients: 26 TAVI and 21 AVR. The preoperative characteristics of the 2 groups were similar with regards to Society of Thoracic Surgeons risk score and comorbidities (Table 1). Patients in the TAVI group were significantly older $(85 \pm 6$ years vs 80 \pm 4 years; $P \leq .001$ ). Overall surgical risk factors were similar between cohorts. The TAVI group did have a significantly higher rate of previous cardiac surgery. All patients with prior cardiac surgery had previously undergone coronary artery bypass surgery, with coronary angiography demonstrating patent mammary artery grafts in each. There was no significant difference between groups when comparing incompletely revascularized coronary territories (TAVI, 8 out of 78 vs AVR, 3 out of $63 ; P=.20$ ). All patients proceeded as clinically indicated to TAVI or AVR intended group.

There was no procedure-related mortality in either group. All patients had a successful procedure and no patient in the TAVI group required conversion to AVR in the first 30 days after the procedure. Mean valve size was larger in the TAVI group $(25.3 \pm 1.8 \mathrm{~mm}$ vs $22.6 \pm 1.9 \mathrm{~mm} ; P<.001)$. In the surgical group the mean cardiopulmonary bypass and crossclamp times were $65 \pm 13$ minutes and $50 \pm 11$ minutes, respectively. Transcatheter patients required between 3 and 4 bursts of rapid ventricular pacing during the procedure. 
TABLE 1. Baseline patient characteristics

\begin{tabular}{|c|c|c|c|}
\hline Characteristic & $\begin{array}{c}\text { Aortic valve } \\
\text { replacement }(n=21)\end{array}$ & $\begin{array}{c}\text { Transcatheter aortic } \\
\text { valve replacement }(n=26)\end{array}$ & $P$ value \\
\hline Age, y & $79.6 \pm 4.0$ & $84.6 \pm 5.6$ & $<.001$ \\
\hline Male & $8(38.1)$ & $17(65.4)$ & .06 \\
\hline Society of Thoracic Surgeons risk score & $5.9 \pm 3.4$ & $7.7 \pm 3.9$ & .11 \\
\hline Brain natriuretic peptide & $1084 \pm 1783$ & $2854 \pm 4944$ & $<.001$ \\
\hline Hypertension & $19(90.5)$ & $24(92.3)$ & .82 \\
\hline Hypercholesterolemia & $15(71.4)$ & $21(80.8)$ & .51 \\
\hline Previous myocardial infarction & $4(19.0)$ & $6(23.1)$ & 1.0 \\
\hline Previous percutaneous coronary intervention & $3(14.3)$ & $8(30.8)$ & .30 \\
\hline Chronic obstructive pulmonary disease & $9(42.9)$ & $11(42.3)$ & .97 \\
\hline Renal impairment & $8(38.1)$ & $9(34.6)$ & .81 \\
\hline Atrial fibrillation & $5(23.8)$ & $6(23.1)$ & .95 \\
\hline Diabetes & $9(42.9)$ & $10(38.5)$ & .76 \\
\hline Redo & - & $11(42.3)$ & $<.001$ \\
\hline Previous cerebrovascular accident/transient ischemic attack & $1(4.8)$ & $9(34.6)$ & .02 \\
\hline Angina & $10(47.6)$ & $8(30.8)$ & .24 \\
\hline New York Heart Association functional class & $2.7 \pm 0.6$ & $2.5 \pm 0.8$ & .14 \\
\hline Pulmonary arterial hypertension & $7(33.3)$ & $5(17.2)$ & .19 \\
\hline Nonrevascularized coronary artery disease & $3(14.3)$ & $8(30.8)$ & .21 \\
\hline
\end{tabular}

Values are presented as mean \pm standard deviation or $\mathrm{n}(\%)$.

\section{CMR Imaging Results}

All patients completed pre- and postprocedure scans. Three patients had inadequate image quality for assessment of aortic valve flow. A total of 47 patients had scans before and after the procedure (100\%) with $44(94 \%)$ having complete imaging of LV/RV volumes, function, and aortic valve flow. Mean time to postoperative scan was $4.7 \pm 4$ days versus $5.8 \pm 2$ days for TAVI and AVR patients, respectively $(P=.14)$.

\section{Change in LV/RV Function After TAVI and AVR}

The CMR changes over time are illustrated in Table 2. LV ejection fraction was preserved postoperatively in both groups (TAVI, $68 \% \pm 12 \%$ vs AVR, $71 \% \pm 13 \%$; $P=.31$ ) with no significant difference in the group $\times$ time interaction $(P=.5)$. There was a significant difference in LV remodeling between TAVI and CMR groups. Despite similar baseline LVEDV $(129 \pm 39 \mathrm{~mL}$ vs $118 \pm 27 \mathrm{~mL}$, respectively; $P=.13$ ), LV dilation was greater following TAVI than AVR (LVEDV, $133 \pm 42 \mathrm{~mL}$ vs $97 \pm 21 \mathrm{~mL}$, respectively; $P<.001$; group $\times$ time interaction, $P<.001)(P<.01)$.

$\mathrm{RV}$ ejection fraction was similar between groups preprocedure (TAVI, $61 \% \pm 11 \%$ vs AVR, $59 \% \pm 8 \%$; $P=$.6). Unlike $\mathrm{LV}$ ejection fraction, RV ejection fraction decreased more in the TAVI group than the AVR group (postprocedure $54 \% \pm 13 \%$ vs $58 \% \pm 8 \% ; P=.1$; group $\times$ time interaction, $P=.008$ ). This difference was driven by differences in RVESV between the groups. Whereas baseline RVESVs were similar between TAVI and AVR groups ( $41 \pm 24 \mathrm{~mL}$ vs $40 \pm 11 \mathrm{~mL} ; P=.8$ ), postprocedure RVESV was significantly larger in the TAVI group $(51 \pm 31 \mathrm{~mL}$ vs $39 \pm 14 \mathrm{~mL}: P=.05$; group $\times$ time interaction, $P=.005$ ). The significant associations between ventricular remodeling/function and procedure type remained significant after adjusting for serum pro brain natriuretic peptide concentration.

Sensitivity analysis was performed to determine whether adjustment for propensity score altered the Cine magnetic resonance imaging results. The LVEDV main effects were unchanged for group $\times$ time interaction $(P=.002)$. The RVejection fraction and RVESV main effects were also unchanged for group $\times$ time interaction $(P=.009$ and $P=.005)$.

\section{Changes to Left Atrial Volume}

Left atrial volume was significantly greater at follow-up in the TAVI group compared with the AVR group (110 \pm $35 \mathrm{~mL}$ vs $84 \pm 24 \mathrm{~mL} ; P=.02$ ). There was a trend toward more favorable reverse remodeling in the AVR group (group $\times$ time interaction, $P=.09$ ).

\section{Changes to Aortic Valve Flow}

Aortic valve flow parameters on CMR imaging are shown in Table 3. Aortic stenosis, as assessed by aortic valve peak velocity and aortic valve area improved over time and were similar between groups. Postprocedure regurgitant fraction revealed significantly greater AR in the TAVI cohort when compared the AVR group (TAVI, $16 \% \pm 16 \%$ vs AVR, $4 \%$ $\pm 2 \% ; P=.005)$.

\section{Myocardial Injury}

Regional wall motion. Thirteen AVR patients $(62 \%)$ and 14 TAVI patients $(54 \%)$ had normal regional wall motion in all segments preoperatively $(P=.56)$. The number of 
TABLE 2. Preoperative and postoperative ventricular function

\begin{tabular}{|c|c|c|c|}
\hline Function & Aortic valve replacement & Transcatheter aortic valve implantation & $P$ for group $\times$ time interaction \\
\hline Left ventricle ejection fraction & & & .48 \\
\hline Preoperative & $73 \% \pm 9 \%$ & $69 \% \pm 13 \%$ & \\
\hline Postoperative & $71 \% \pm 13 \%$ & $68 \% \pm 12 \%$ & \\
\hline Left ventricular end-diastolic volume, $\mathrm{mL}$ & & & $<.01$ \\
\hline Preoperative & $118 \pm 27$ & $129 \pm 39$ & \\
\hline Postoperative & $97 \pm 21$ & $133 \pm 42$ & \\
\hline Left ventricular end-systolic volume, $\mathrm{mL}$ & & & .54 \\
\hline Preoperative & $33 \pm 15$ & $43 \pm 28$ & \\
\hline Postoperative & $30 \pm 17$ & $44 \pm 32$ & \\
\hline Right ventricle ejection fraction & & & .01 \\
\hline Preoperative & $59 \% \pm 8 \%$ & $61 \% \pm 11 \%$ & \\
\hline Postoperative & $58 \% \pm 8 \%$ & $54 \% \pm 13 \%$ & \\
\hline Right ventricular end-diastolic volume, $\mathrm{mL}$ & & & .28 \\
\hline Preoperative & $98 \pm 22$ & $104 \pm 37$ & \\
\hline Postoperative & $95 \pm 27$ & $108 \pm 42$ & \\
\hline Right ventricular end-systolic volume, $\mathrm{mL}$ & & & $<.01$ \\
\hline Preoperative & $40 \pm 11$ & $41 \pm 24$ & \\
\hline Postoperative & $39 \pm 14$ & $51 \pm 31$ & \\
\hline
\end{tabular}

Values are presented as mean \pm standard deviation.

patients in each group with 3 or more dysfunctional segments preprocedure was also similar (TAVI 35\% and AVR 29\%; $P=.65)$. In addition, there was no difference in the change in segmental wall motion between groups (group $\times$ time interaction, $P=.42$ for wall motion score index).

Late gadolinium enahancement imaging. LGE imaging was obtained preprocedure in $81 \%$ and $86 \%(P=.47)$ and postprocedure in $76 \%$ and $86 \%(P=.73)$ of AVR and TAVI patients, respectively. Preoperatively 9 TAVI patients $(45 \%)$ and 5 AVR patients $(33 \%)$ demonstrated LGE in at least $1 \mathrm{LV}$ slice $(P=.49)$. Postoperatively there was new LGE in 2 TAVI patients $(11 \%)$ and 2 AVR patients $(13 \%)(P=.72)$.

\section{Diastolic LV Function}

The E/E' ratio-an index of diastolic LV functionshowed a significant reduction in the AVR group compared

TABLE 3. Aortic valve flow

\begin{tabular}{lccc}
\hline \multicolumn{1}{c}{ Variable } & $\begin{array}{c}\text { Aranscatheter } \\
\text { replacement }\end{array}$ & $\begin{array}{c}\boldsymbol{P} \text { for } \\
\text { aortic valve } \\
\text { implantation }\end{array}$ & $\begin{array}{c}\text { group } \times \text { time } \\
\text { interaction }\end{array}$ \\
\hline $\begin{array}{l}\text { Forward volume, } \mathrm{mL} \\
\quad \text { Preoperative }\end{array}$ & $59 \pm 24$ & $57 \pm 22$ & .07 \\
$\quad \begin{array}{l}\text { Postopoperative } \\
\text { Regurgitant fraction }\end{array}$ & $66 \pm 24$ & $76 \pm 26$ & \\
$\quad$ Preoperative & $12 \% \pm 16 \%$ & $18 \% \pm 15 \%$ & .51 \\
$\quad \begin{array}{l}\text { Postoperative } \\
\text { Peak velocity, } \mathrm{m} / \mathrm{s}\end{array}$ & $4.0 \% \pm 2.4 \%$ & $16 \% \pm 16 \%$ & \\
$\quad$ Preoperative & $3.6 \pm 0.81$ & $3.9 \pm 1.0$ & .71 \\
$\quad$ Postoperative & $2.6 \pm 0.62$ & $2.4 \pm 0.49$ & \\
Aortic valve area, $\mathrm{cm}^{2}$ & & & .12 \\
$\quad$ Preoperative & $0.74 \pm 0.18$ & $0.83 \pm 0.21$ & \\
Postoperative & $1.8 \pm 0.40$ & $1.7 \pm 0.29$ & \\
\hline
\end{tabular}

Values are presented as mean \pm standard deviation. with the TAVI patients (baseline $\mathrm{E} / \mathrm{E}^{\prime}$ in the AVR group was $23 \pm 9$ vs $23 \pm 8$ in the TAVI group $[P=.4]$; postprocedure $\mathrm{E} / \mathrm{E}^{\prime} 19 \pm 7$ in the AVR group vs $23 \pm 7$, in the TAVI group $[P=.05]$; group-time interaction $[P=.03])$.

\section{Post Hoc Analyses}

Following the finding of right ventricular dysfunction in the TAVI cohort, ancillary statistical analyses assessed for influences on the change in RV function. There was a trend toward a negative association between regurgitant fraction and RV ejection fraction (coefficient, $-0.10 ; 95 \%$ confidence interval, -0.22 to $0.011 ; P=.08$ ) (Figure 2). To further assess this finding the TAVI cohort was divided into those with mild or less paravalvular aortic regurgitation (PAR) and those with moderate or greater PAR. There was no significant difference in preprocedure $\mathrm{RV}$ ejection fraction between groups $(\leq$ mild $62 \% \pm 10 \%$ vs $\geq$ moderate $59 \% \pm 13 \% ; P=.16$ ). Postprocedure there was significantly worse RV ejection fraction in those patients with moderate or greater PAR $(\leq$ mild $58 \%$ $\pm 11 \%$ vs $\geq$ moderate $48 \% \pm 13 \% ; P=.03$ ). Additionally there was a correlation between change in aortic valve regurgitant fraction and left atrial dilation postprocedure (coefficient, $2.65 ; 95 \%$ confidence interval, $0.75-4.55 ; P=.006$ ), although we were unable to show a significant association between left atrial volume and RV ejection fraction. Given the potential for ischemia-induced RV dysfunction further analysis of the angiographic QCA data were sought. These demonstrated no difference in the number of incompletely revascularized territories $(P=.91)$.

\section{DISCUSSION}

The principal findings from our study are that $\mathrm{LV}$ ejection fraction was equally well preserved in both TAVI and AVR 


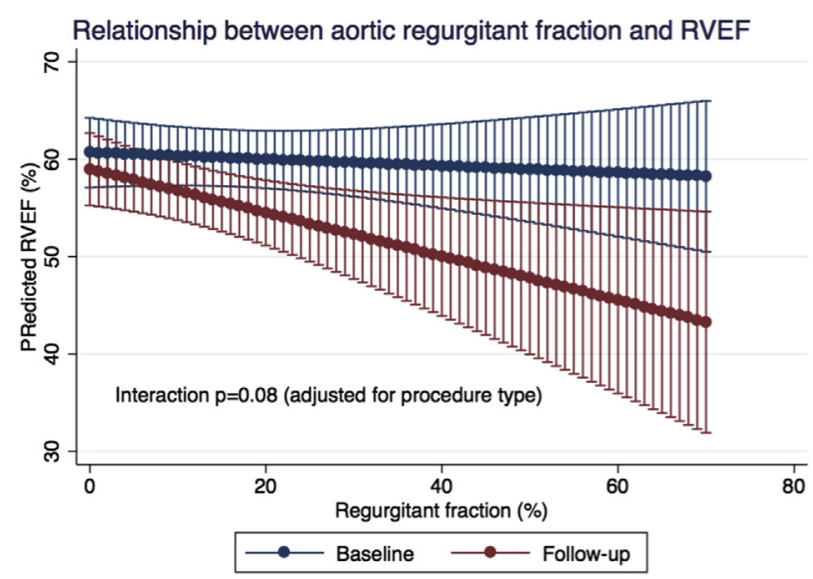

FIGURE 2. Graphic representation of the relationship between aortic regurgitation assessed by cardiovascular magnetic resonance imaging and right ventricle ejection fraction (RVEF) assessed by cardiovascular magnetic resonance imaging.

groups early following the procedure and that RV systolic function was impaired early after TAVI, and potentially associated with PAR. Given the prognostic importance of RV dysfunction, our findings provide a potential mechanism for the recent observation of increased mortality in the setting of post-TAVI AR.

No previous study has used CMR imaging for serial assessment and comparison of perioperative $(<14$ days $)$ myocardial effects of AVR and TAVI. Previous CMRbased studies have conducted scans at preprocedure and 6 months postprocedure offering a midterm outlook. ${ }^{26,27}$ Our study findings do not support the primary hypothesis that LV function would be better preserved by TAVI when compared with AVR. Imaging at similar intervals postprocedure revealed no significant difference in global left ventricular systolic function (ie, LV ejection fraction). Indeed, volumetric measures of LV remodeling favored AVR. Ewe and colleagues ${ }^{28}$ used TTE to assess early ( $\leq 48$ hours) postprocedure LV global function in 147 patients undergoing TAVI and compared this to a retrospective surgical control group of 99 patients. These investigators reported no significant change in LV ejection fraction in patients with baseline LV ejection fraction $>50 \%$ who underwent TAVI. ${ }^{28}$ Earlier work by Clavel and colleagues ${ }^{29}$ in a TAVI population with matched surgical controls revealed similar findings with no significant difference or change in LV function. ${ }^{29}$ The majority of patients in our cohort had normal $(>55 \%) \mathrm{LV}$ ejection fraction preprocedure $(85 \%$ of TAVI and $95 \%$ of AVR patients). Hence, despite the use of a more sensitive and reproducible technique (ie, CMR), our findings on global LV function support previous echocardiographic studies. Our hypothesis was based on an expected relative decrease in LV ejection fraction in the AVR group secondary to the ischemic insult of cardiopulmonary bypass and aortic crossclamping. Our results suggest that there is no left ventricular functional benefit of a transcatheter approach despite the absence of ischemic insult.

There were differences in LV volumetric indices between the 2 groups, but contrary to our initial hypothesis, this favored the AVR group. Postoperative LVEDV demonstrated a trend favoring the AVR group (Figure 3) and this reduction was partially matched by a nonsignificant reduction in LVESV in the AVR group accounting for the lack of change in LV ejection fraction. The presence of reduced postoperative LVEDV can be explained simply by the reduced outflow obstruction leading to a lower end-diastolic pressure and subsequently reduced enddiastolic volume. Successful resolution of aortic stenosis would be expected to have this effect in both groups; however, this was not seen in the TAVI group. We propose this may be an effect of acutely increased postprocedure AR increasing end-diastolic pressure. This may offset the benefit that aortic valve obstruction resolution provides and result in static LV volumetric measures after TAVI. Previous echocardiographic studies in TAVI cohorts have not shown any significant reduction in LVEDV (or LV end-diastolic diameter) early postprocedure in either normal or impaired LV ejection fraction. ${ }^{28,30}$ The next generation of TAVI devices may confirm the mechanism of volumetric changes post TAVI should they decrease the incidence and severity of postprocedure AR.

Despite the fact that both groups had preserved RV function at baseline, there was significantly greater RV dysfunction in the TAVI group. RV ejection fraction decreased significantly in TAVI patients compared with the AVR patients, driven largely by an increase in RVESV in TAVI patients. The RV is recognized as a thin-walled, highly compliant structure with a primary compensatory mechanism to injury-irrespective of the cause-of dilation. ${ }^{31}$ The AVR cohort demonstrated a decrease in both RVEDV and RVESV. To the best of our knowledge this study is the first to report a decrease in RV ejection fraction among TAVI patients when compared with AVR and tellingly the only report of CMR-derived RV parameters. None of the large trials or registries has focused on RV function and several small echocardiographic studies did not report significant changes. ${ }^{7,32}$ Kempny and colleagues $^{33}$ and Zhao and colleagues ${ }^{34}$ had findings contradictory to ours with echocardiography assessed RV function preserved in a TAVI cohort and decreased in a surgical control group. The discrepancy between findings is likely explained by several methodologic limitations, namely unmatched baseline characteristics, mixture of mechanical and bioprosthetic valves, mixture of transfemoral and transapical valves, and concomitant bypass surgery in the surgical group. Moreover, the echocardiographic studies reported elsewhere largely used secondary measures of RV function such as tricuspid annular plane systolic excursion 

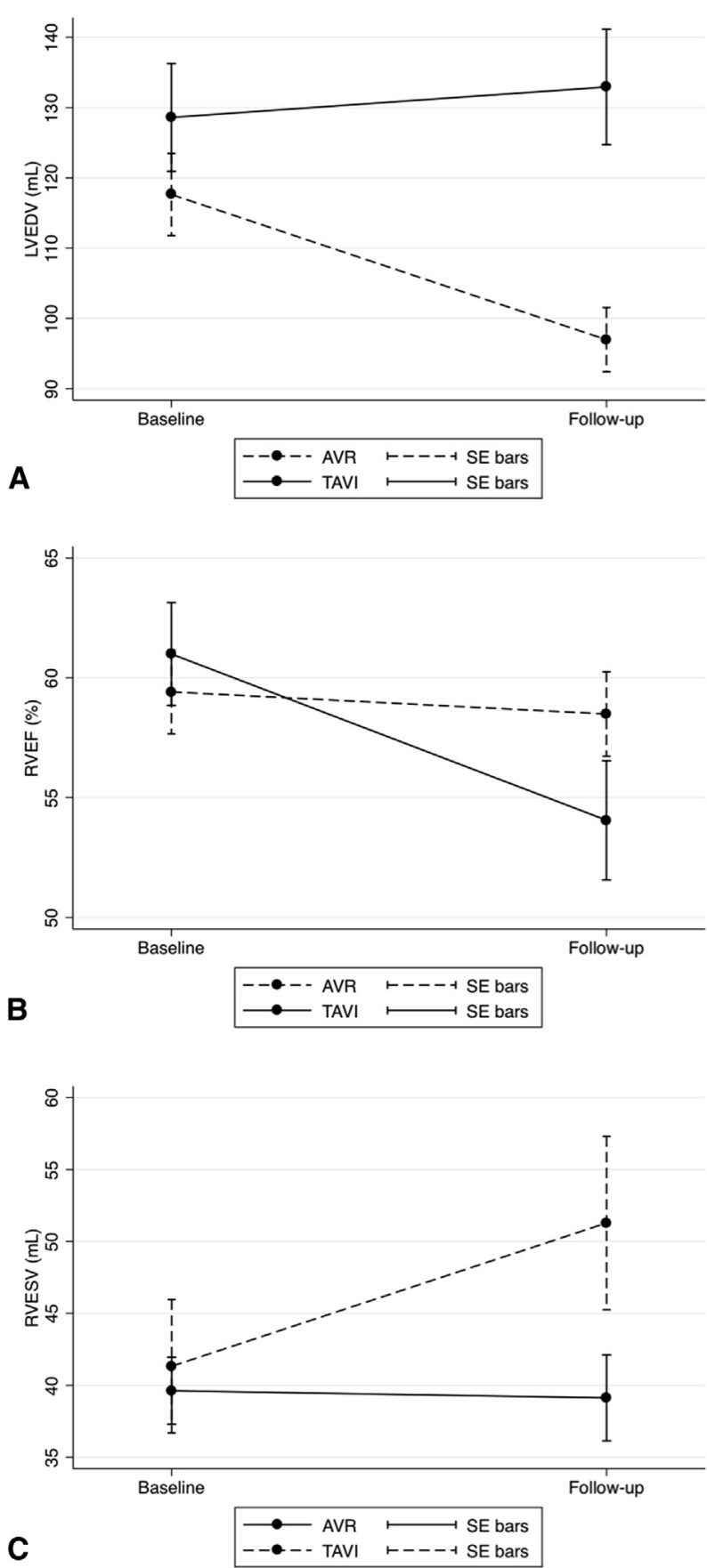

FIGURE 3. Differential effects of open aortic valve replacement $(A V R)$ and transcatheter aortic valve implantation $(T A V I)$ on indices of cardiac remodeling and function. A, left ventricular end-diastolic volume $(L V E D V)$. B. Right ventricle ejection fraction $(R V E F)$. C, Right ventricular end-systolic volume (RVESV). SE, Standard error.

and RV strain; these are inherently difficult to assess particularly in a surgical population owing to difficult image acquisition and variances induced by pericardiotomy and septal function. Furthermore the secondary nature of measurements such as tricuspid annular plane systolic excursion mean that whereas tricuspid excursion may change postprocedure, adaptive mechanisms may maintain RV ejection fraction. ${ }^{11}$ Finally the increased accuracy of CMR RV interrogation over echocardiographic imaging is well recognized.

Even after propensity score adjustment and statistical reevaluation to account for the difference in $\mathrm{N}$-terminal pro B-type natriuretic peptide, we found a significant decrease in RV ejection fraction in the TAVI cohort compared with those undergoing AVR. We thought the most likely etiology of this dysfunction was the increased incidence and severity of PAR in the TAVI group. This hypothesis was supported by ancillary analysis revealing 2 key findings. The first was a trend correlation between decreased postprocedure RV ejection fraction and increased aortic regurgitant fraction $(P=.08)$. The second was the finding of significantly worse RV ejection fraction in those TAVI patients with moderate or greater PAR (as assessed by CMR) compared with those with mild or less PAR. The differences in between-group postprocedure $\mathrm{E} / \mathrm{E}^{\prime}$ and $\mathrm{E} / \mathrm{E}^{\prime}$ group $\times$ time interaction, suggested a mechanism whereby increased left-sided filling pressures secondary to PAR potentially leading to an increased load on the right ventricle. Forsberg and colleagues ${ }^{32}$ also found $E / E^{\prime}$ measurements suggested high LV filling pressures in a TAVI cohort compared with surgical AVR. The correlation of increased PAR to increased left atrial volume, both of which were significantly worse in the TAVI group, further supports a potential mechanism for our novel finding. We postulate that the AR associated with TAVI rather than AVR results in increased LV end-diastolic pressure (the hypertrophic ventricle being less compliant), increasing left atrial pressure $\left(\mathrm{E} / \mathrm{E}^{\prime}\right)$ and volume (left atrial volume) leading to venous/passive pulmonary hypertension that increases strain on the precariously positioned RV leading to reduced RV ejection fraction. Given the adverse clinical consequences of RV dysfunction in the syndrome of heart failure, this chain of events resulting in RV dysfunction offers a potential hypothesis for the important clinical observation of increased mortality in patients with AR post-TAVI. This hypothesis can be further investigated with medium- and long-term data examining both RV function and clinical outcomes. Another potential cause of RV dysfunction-ischemia-is unlikely given the lack of difference on coronary QCA.

Overall the irreversible myocardial injury rate was low in both groups. Our findings did not support our hypothesis that there would be a significant rate of irreversible injury in both groups. Even with a higher incidence of unrevascularized coronary artery disease in the TAVI group injury rates were similar. Despite using the highly sensitive late enhancement CMR imaging technique, we found that only $8 \%$ of TAVI and $10 \%$ of AVR patients demonstrated new irreversible myocardial injury (ie, type V acute myocardial infarction). ${ }^{35}$ The Placement of Aortic 
Transcatheter Valves (PARTNER) trial reported infarct rates of $0 \%$ and $0.6 \%$ for TAVI and AVR, respectively, using clinical and biochemical data; and the higher rate reflects the higher sensitivity of the CMR technique. The clinical significance of these small areas of myocardial injury is uncertain, although our previous work in a population undergoing coronary artery bypass grafting indicates that it may not be entirely benign. ${ }^{3}$

\section{Limitations}

The most significant limitation of this trial is the nonrandomized design. Regulatory approval limitations mean TAVI remains utilized largely in the inoperable cohort, limiting randomization. As expected, the TAVI group members were significantly older because they consisted of both technically and medically high-risk patients. The higher rate of reoperative cases in the TAVI group was attributable to the evolution of TAVI as the preferred treatment for patients with patent bypass grafts and multiple comorbidities. The question of increased susceptibility to periprocedural ischemic injury in these patients was addressed with the findings that neither QCA or regional wall motion abnormality assessment demonstrated differences between groups. Despite the difference in age and redo status, the groups were matched regarding Society of Thoracic Surgeons risk score, as they were for CMR-derived preprocedure cardiac indices. Moreover, our attempt to adjust for risk factors using propensity score and post-hoc mixed effects regression modeling demonstrated no change in the significant findings. Overall the 2 groups were sufficiently matched and post-hoc analysis was sufficiently robust that results seen in this study reflect the effects of procedural technique rather than preexisting patient characteristics.

The small cohort numbers is also an obvious limitation. One of the inherent advantages of CMR over echocardiography or other modalities is the accuracy and reduced observer variability it provides, allowing smaller research cohorts. Although the ability to postulate a link between $\mathrm{AR}$ and RV dysfunction is hampered by our small numbers, we believe this new observation warrants focused study in a future mechanistic study. Finally, the inherent effects of cardiovascular loading states need to be considered. Patients underwent scanning during the early morning and all were at least 48 hours postprocedure with mean time of 6 to 7 days. The similarity in time of day and time postprocedure to allow for settling of fluid shifts we believe minimizes the variation from cardiovascular loading. This would minimize the influence on factors such as left atrial volume. We do acknowledge the difficulties in measuring left atrial volume, regardless of the methodology.

\section{CONCLUSIONS}

Patients undergoing either AVR or TAVI have preserved LV function after the procedure. Furthermore although irreversible injury rates were very low in both groups, they are higher than has been reported using clinical and/ or biochemical methods in large trials.

Our results demonstrate for the first time that TAVI is associated with early RV dysfunction. This may reflect the higher incidence of AR with TAVI and explain the recent observation of increased long-term mortality in this setting.

The authors thank Suchi Grover for assistance with the secondary CMR analysis.

\section{References}

1. Dweck MR, Boon NA, Newby DE. Calcific aortic stenosis: a disease of the valve and the myocardium. J Am Coll Cardiol. 2012;60:1854-63.

2. Beckmann A, Hamm C, Figulla HR, Cremer J, Kuck KH, Lange R, et al. The German Aortic Valve Registry (GARY): a nationwide registry for patients undergoing invasive therapy for severe aortic valve stenosis. Thorac Cardiovasc Surg. 2012;60:319-25

3. Makkar RR, Fontana GP, Jilaihawi H, Kapadia S, Pichard AD, Douglas PS, et al Transcatheter aortic-valve replacement for inoperable severe aortic stenosis. $N$ Engl J Med. 2012;366:1696-704.

4. Onorati F, D'Errigo P, Grossi C, Barbanti M, Ranucci M, Covello DR, et al. Effect of severe left ventricular systolic dysfunction on hospital outcome after transcatheter aortic valve implantation or surgical aortic valve replacement: results from a propensity-matched population of the Italian OBSERVANT multicenter study. J Thorac Cardiovasc Surg. 2014;147:568-75.

5. Smith CR, Leon MB, Mack MJ, Miller DC, Moses JW, Svensson LG, et al. Transcatheter versus surgical aortic-valve replacement in high-risk patients. $N$ Engl J Med. 2011;364:2187-98.

6. Tice JA, Sellke FW, Schaff HV. Transcatheter aortic valve replacement in patients with severe aortic stenosis who are at high risk for surgical complications: summary assessment of the California Technology Assessment Forum. J Thorac Cardiovasc Surg. 2014;148:482-91.e6.

7. Kodali SK, Williams MR, Smith CR, Svensson LG, Webb JG, Makkar RR, et al Two-year outcomes after transcatheter or surgical aortic-valve replacement. $N$ Engl J Med. 2012;366:1686-95.

8. Bondarenko O, Beek AM, Nijveldt R, McCann GP, van Dockum WG, Hofman MB, et al. Functional outcome after revascularization in patients with chronic ischemic heart disease: a quantitative late gadolinium enhancement CMR study evaluating transmural scar extent, wall thickness and periprocedural necrosis. J Cardiovasc Magn Reson. 2007;9:815-21.

9. Selvanayagam JB, Petersen SE, Francis JM, Robson MD, Kardos A, Neubauer S, et al. Effects of off-pump versus on-pump coronary surgery on reversible and irreversible myocardial injury: a randomized trial using cardiovascular magnetic resonance imaging and biochemical markers. Circulation. 2004;109:345-50.

10. Goncalves A, Marcos-Alberca P, Almeria C, Feltes G, Rodriguez E, HernandezAntolin RA, et al. Acute left ventricle diastolic function improvement after transcatheter aortic valve implantation. Eur J Echocardiogr. 2011;12:790-7.

11. Lindqvist P, Calcutteea A, Henein M. Echocardiography in the assessment of right heart function. Eur J Echocardiogr. 2008;9:225-34.

12. Pegg TJ, Selvanayagam JB, Karamitsos TD, Arnold RJ, Francis JM, Neubauer S, et al. Effects of off-pump versus on-pump coronary artery bypass grafting on early and late right ventricular function. Circulation. 2008;117:2202-10.

13. Pegg TJ, Maunsell Z, Karamitsos TD, Taylor RP, James T, Francis JM, et al. Utility of cardiac biomarkers for the diagnosis of type $\mathrm{V}$ myocardial infarction after coronary artery bypass grafting: insights from serial cardiac MRI. Heart. 2011; 97:810-6.

14. Selvanayagam JB, Kardos A, Francis JM, Wiesmann F, Petersen SE, Taggart DP, et al. Value of delayed-enhancement cardiovascular magnetic resonance imaging in predicting myocardial viability after surgical revascularization. Circulation. 2004; 110:1535-41.

15. Selvanayagam JB, Porto I, Channon K, Petersen SE, Francis JM, Neubauer S et al. Troponin elevation after percutaneous coronary intervention directly represents the extent of irreversible myocardial injury: insights from cardiovascular magnetic resonance imaging. Circulation. 2005;111:1027-32.

16. Selvanayagam JB, Cheng AS, Jerosch-Herold M, Rahimi K, Porto I, van Gaal W, et al. Effect of distal embolization on myocardial perfusion reserve after 
percutaneous coronary intervention: a quantitative magnetic resonance perfusion study. Circulation. 2007;116:1458-64.

17. Chandran KB, Udaykumar HS, Reinhardt JM. Image-based computational modeling of the human circulatory and pulmonary systems: methods and applications. Iowa City, IA: Springer; 2010.

18. Pennell DJ. Ventricular volume and mass by CMR. J Cardiovasc Magn Reson. 2002;4:507-13.

19. Pennell DJ. Cardiovascular magnetic resonance. Circulation. 2010;121:692-705.

20. Myerson SG. Heart valve disease: investigation by cardiovascular magnetic resonance. J Cardiovasc Magn Reson. 2012;14:7.

21. Michel P, Roques F, Nashef SA. Logistic or additive EuroSCORE for high-risk patients? Eur J Cardiothorac Surg. 2003;23:684-7; discussion 687.

22. Leong DP, Chakrabarty A, Shipp N, Molaee P, Madsen PL, Joerg L, et al. Effects of myocardial fibrosis and ventricular dyssynchrony on response to therapy in new-presentation idiopathic dilated cardiomyopathy: insights from cardiovascular magnetic resonance and echocardiography. Eur Heart J. 2012;33:640-8.

23. Lang RM, Bierig M, Devereux RB, Flachskampf FA, Foster E, Pellikka PA, et al. Recommendations for chamber quantification. Eur J Echocardiogr. 2006;7: 79-108.

24. Amado LC, Gerber BL, Gupta SN, Rettmann DW, Szarf G, Schock R, et al. Accurate and objective infarct sizing by contrast-enhanced magnetic resonance imaging in a canine myocardial infarction model. J Am Coll Cardiol. 2004;44:2383-9.

25. Matthews JN, Altman DG, Campbell MJ, Royston P. Analysis of serial measurements in medical research. BMJ. 1990;300:230-5.

26. Fairbairn TA, Steadman CD, Mather AN, Motwani M, Blackman DJ, Plein S, et al. Assessment of valve haemodynamics, reverse ventricular remodelling and myocardial fibrosis following transcatheter aortic valve implantation compared to surgical aortic valve replacement: a cardiovascular magnetic resonance study. Heart. 2013;99:1185-91.

27. La Manna A, Sanfilippo A, Capodanno D, Salemi A, Cadoni A, Cascone I, et al. Left ventricular reverse remodeling after transcatheter aortic valve implanta- tion: a cardiovascular magnetic resonance study. J Cardiovasc Magn Reson. 2013;15:39.

28. Ewe SH, Ajmone Marsan N, Pepi M, Delgado V, Tamborini G, Muratori M, et al. Impact of left ventricular systolic function on clinical and echocardiographic outcomes following transcatheter aortic valve implantation for severe aortic stenosis. Am Heart J. 2010;160:1113-20.

29. Clavel MA, Webb JG, Pibarot P, Altwegg L, Dumont E, Thompson C, et al. Comparison of the hemodynamic performance of percutaneous and surgical bioprostheses for the treatment of severe aortic stenosis. J Am Coll Cardiol. 2009; 53:1883-91.

30. Kempny A, Diller GP, Kaleschke G, Orwat S, Funke A, Schmidt R, et al. Impact of transcatheter aortic valve implantation or surgical aortic valve replacement on right ventricular function-the reply. Heart. 2012;98:1299-304.

31. Sidebotham D. Cardiothoracic critical care. Philadelphia: Butterworth-Heinemann; 2007.

32. Forsberg LM, Tamas E, Vanky F, Nielsen NE, Engvall J, Nylander E. Left and right ventricular function in aortic stenosis patients 8 weeks post-transcatheter aortic valve implantation or surgical aortic valve replacement. Eur J Echocardiogr. 2011;12:603-11.

33. Kempny A, Diller GP, Kaleschke G, Orwat S, Funke A, Schmidt R, et al. Impact of transcatheter aortic valve implantation or surgical aortic valve replacement on right ventricular function. Heart. 2012;98:1299-304.

34. Zhao Y, Lindqvist P, Nilsson J, Holmgren A, Naslund U, Henein MY. Trans-catheter aortic valve implantation-early recovery of left and preservation of right ventricular function. Interact Cardiovasc Thorac Surg. 2011;12:35-9.

35. Thygesen K, Alpert JS, Jaffe AS, Simoons ML, Chaitman BR, White HD Third universal definition of myocardial infarction. Eur Heart J. 2012;33: 2551-67.

36. Rahimi K, Banning AP, Cheng AS, Pegg TJ, Karamitsos TD, Channon KM, et al. Prognostic value of coronary revascularisation-related myocardial injury: a cardiac magnetic resonance imaging study. Heart. 2009;95:1937-43. 